Ann. Zootech., I980, 29 (3), 265-276.

\title{
La production de foie gras de canard de Barbarie mâle : analyse de différents facteurs de variation
}

\author{
R. BABILE ( $\left.{ }^{1}\right)$, G. MATHERON ( $\left.{ }^{2}\right)$, P. DELPECH $\left({ }^{3}\right)$ et M. T. FARRAN (1)
}

\author{
(1) Laboratoive de Zootechnie et des Productions Animales, \\ École Nationale Supérieuve Agronomique, \\ I45, avenue de Muvet, 3Io76 Toulouse Cédex (France) \\ (') Station d'Amélioration Génétique des Animaux, \\ Centre de Recherche de Toulouse-Auzeville, I.N.R.A., \\ chemin de Borde-Rouge, Auzeville, 31320 Castanet Tolosan (France) \\ (3) Institut National Agronomique Paris-Grignon \\ Chaive de Zootechnie, Centre de Grignon, \\ 78850 Thivernal-Grignon (France)
}

\begin{abstract}
Résumé
Un essai est entrepris afin de comparer différents types génétiques de canards de Barbarie mâles ( 2 souches pures, un croisement commercial et un croisement expérimental) pour la production de foie gras. Tous les animaux élevés simultanément ont été contrôlés à différents stades de développement jusqu'à $\mathrm{I} 2$ semaines et après gavage dants un même atelier avec deux gaveurs. L'analyse statistique des résultats met en évidence un effet significatif du type génétique sur tous les critères mesurés, un effet significatif du gaveur sur les critères économiques de gavage et un effet classification des foies lié au poids des animaux à partir d'un certain âge ( 8 semaines) et aux performances de gavage. Les poids de carcasse et de foje du croisement étudié sont comparables à ceux obtenus pour la souche commercialisée. Ce type gérétique présente cependant unie fottte de foie importante après étuvage. Ces résultats nous laissent supposer la nécessité de préciser le mode d'emploi de chacune des souches commercialisables pour en tirer le meilleur parti.
\end{abstract}

\section{Introduction}

Technique artisanale et traditionnelle par excellence, le gavage des palmipèdes, pratiqué surtout dans le sud-ouest de la France, semble connaître, depuis quelques années, un nouvel essor se traduisant par une organisation progressive de la profession. Environ 65 p. Ioo de la production de foie gras provient du gavage des canards (Mulard et Barbarie). 
Pour une meilleure connaissance des souches de canard de Barbarie (Cairina Moschata) destinés à la production de foie gras, nous avons été conduit, en collaboration avec des producteurs, à réaliser une série d'expérimentations (FARRAN, I978; BABILE, I980).

Iors d'une première étude, nous avons comparé différents types génétiques (FARRAN, I978). Les résultats obtenus ont permis de mettre en évidence la supériorité, sur le plan des qualités de croissance et de carcasse, d'une souche pure actuellement utilisée dans un croisement commercial (Grimaud Frères, S.A.R.L.). Lors de cette expérience nous n'avons remarqué aucune différence significative en ce qui concerne le poids des foies. Cependant, une souche pure de petit format a présenté, dans cette étude, un rendement en foie supérieur aux autres types génétiques mis en comparaison pour une qualité de foie équivalente. Cet ensemble de résultats nous a conduit à mettre en place une expérimentation où le croisement entre ces deux souches serait étudié pour son aptitude à l'élevage et au gavage et comparé au type commercial.

\section{Matériel et méthodes}

\section{Animaux et régimes}

Les canards mâles utilisés produits par la Société Grimaud Frères se répartissent en quatre lots de Ioo individus chacun :

- lot II : souche pure de grand format dont nous avons mis en évidence les qualités de carcasse lors de l'expérimentation précédente (FARRAN, I978);

- lot 22 : souche pure de petit format présentant un rendement en foie exceptionnel (FARRAN, I978);

- lot I2: croisement entre des mâles I i et des femelles 22.

- lot I3 : croisement actuellement commercialisé et servant de référence.

Tous ces animaux sont issus d'une même incubation (novembre I978) et bagués à la naissance sur les deux ailes. L'élevage a été réalisé au Centre de Grignon (région parisienne) jusqu'à ce que les canetons atteignent l'âge de I 2 semaines, en cases sur caillebotis grillagés $(2 \mathrm{~m} \times 2 \mathrm{~m})$ de 30 animaux de même type génétique. La répartition des lots dans le bâtiment est aléatoire. La prophylaxie est celle classiquement réalisée dans les élevages (FARRAN, I978). L'aliment commercial distribué ad libitum jusqu'à 1'âge de I2 semaines, a la composition suivante en fonction de l'âge des animaux (tabl. I).

L_es formules sont à base de maïs, mélasse, issues de céréales, tourteau de soja. L'aliment " finition " a en plus de la farine de luzerne déshydratée. Les canetons mâles sont transportés à I 2 semaines, à l'aide d'un camion spécialement aménagé, chez un couple de gaveurs, parmi les meilleurs adhérents du GAPYVOL (Groupement Agricole Pyrénées Volailles) dans le Gers (Samatan).

Un aliment prégavage est distribué pendant trois jours, il contient 7o,8 p. Ioo de maïs, Io p. Ioo de féveroles, I 5 p. I oo de tourteau de soja et 4,2 p. Ioo d'un complément minéral et vitaminique auquel sont ajoutés des ferments lactiques. 


\section{TABIEAU I}

Caractéristiques de l'aliment commercial distribué en fonction de l'âge des animaux Characteristios of the commerctal feed given to the ducks according to their age

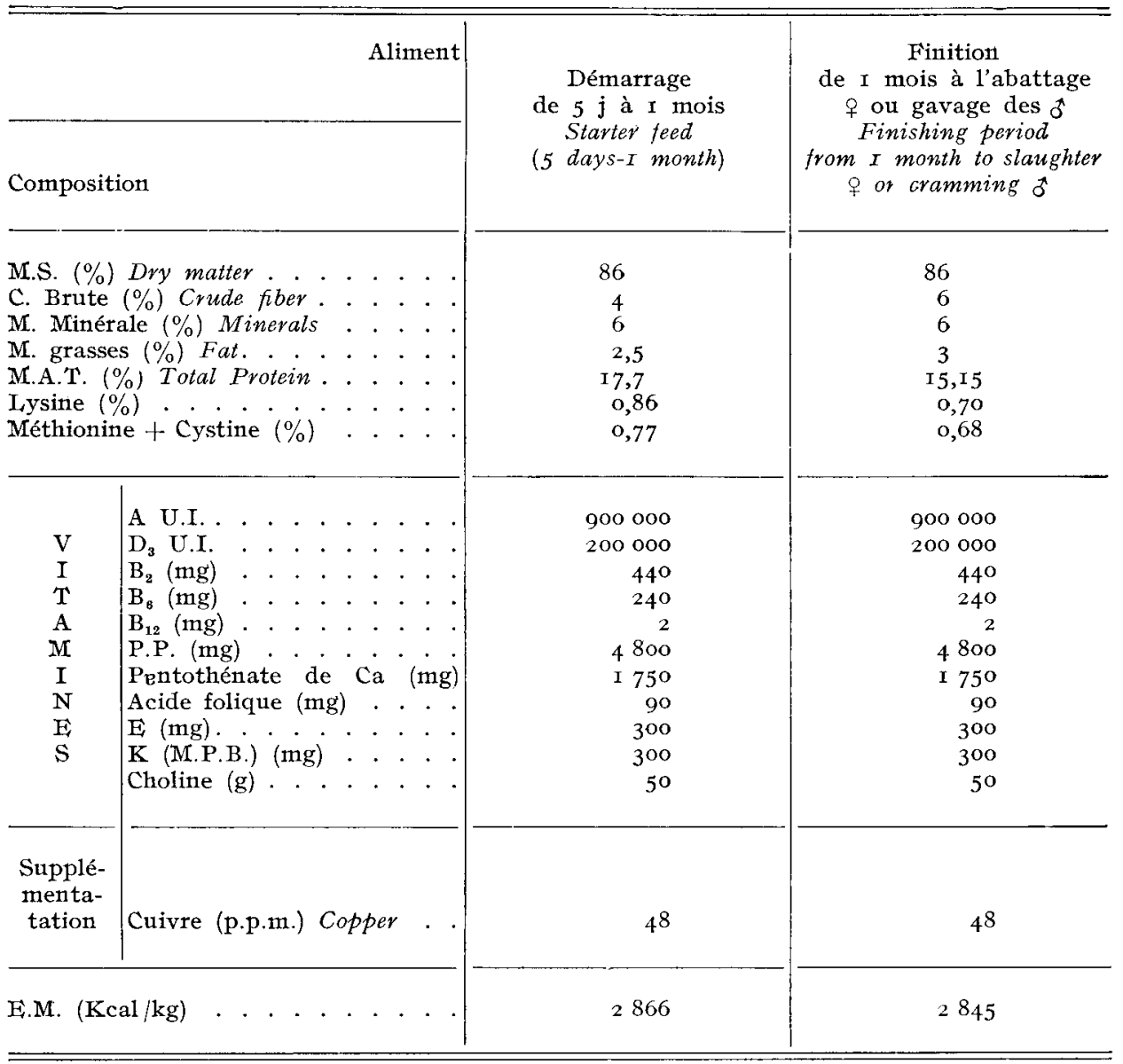

Ensuite les animaux sont gavés au sol sur litière en I6 parquets de 20 à 25 canards en début de bande, chaque gaveur ayant 8 parquets à sa charge. Les parquets sont constitués d'animaux de même type génétique et répartis équitablement entre les deux gaveurs de l'atelier. Les animaux sont gavés deux fois par jour avec un mélange de maïs blanc et jaune précuit, additionné de matières grasses et de sel. Les proportions et quantités sont déterminées par le gaveur. L'effet de ce mode de conduite inclu dans nos résultats par la prise en compte de l'effet gaveur sera détaillé dans une publication ultérieure.

Les dates d'abattage ont été laissées au libre choix des gaveurs et se sont étalées en fonction de l'état de finition des animaux âgés à ce stade đe I 5 semaines \pm 3 jours. 


\section{Caractères mesurés}

Des pesées individuelles faites le matin sur des animaux à jeûn ont été réalisées durant toute la période d'élevage $(5 \mathrm{j}, 25 \mathrm{j}, 53 \mathrm{j}, 67 \mathrm{j}, 8 \mathrm{r}$ j). Les animaux sont toujours pris dans le même ordre. Après gavage, les poids de carcasse (saigné plumé), de foie et de paletots ont été enregistrés, de même que le classement des foies selon les normes de qualité établies par la S.I.C.A. St-Sever (tab1. 2). Sur chaque foie un échantillon de quelques grammes a été prélevé (pointe du lobe intermédiaire gauche) (PAVAux et Jolıy, I968). Ces échantillons pesés avant et après étuvage à $55^{\circ}$ puis à $105^{\circ} \mathrm{C}$, donnent une mesure de la fonte du foie à $1^{\prime}$ issue de chacun de ces deux traitements par rapport au poids frais. Nous avons extrapolé le pourcentage de fonte observé sur le lobule prélevé à l'ensemble du foie pour obtenir une valeur de fonte chiffrée en grammes du foie entier. Les échantillons ont été conservés par congélation avant les traitements par étuvage.

\section{TABLEAU 2}

Critères de classifucation des foies de canard Criteria used for liver classification

\begin{tabular}{|c|c|c|c|c|}
\hline $\begin{array}{l}\text { Qualité } \\
\text { (Quality) }\end{array}$ & $\begin{array}{l}\text { Poids } \\
(\text { Weight })\end{array}$ & $\begin{array}{l}\text { Apparence } \\
\quad(A s p e c t)\end{array}$ & Texture & $\begin{array}{l}\text { Odeur } \\
\text { (Odour) }\end{array}$ \\
\hline Extra A & $\begin{array}{l}\text { Minimum } \\
400 \mathrm{~g}\end{array}$ & $\begin{array}{l}\text { * Paroi extérieure lisse, blanc-rosé, } \\
\text { couleur uniforme, sans tâches rou- } \\
\text { geâtres, trace sanguine ou défaut } \\
\text { apparent. }\end{array}$ & \multirow{2}{*}{$\begin{array}{l}\text { Ferme, mais } \\
\text { souple } \\
\text { au toucher }\end{array}$} & \multirow{4}{*}{$\begin{array}{l}\text { Sans odeur } \\
\text { prononcée } \\
\text { (Without } \\
\text { any marked } \\
\text { odour) }\end{array}$} \\
\hline Fixtra B & $\begin{array}{l}\quad \text { Sans } \\
\text { distinction } \\
\text { (Withont } \\
\text { distinction) }\end{array}$ & $\begin{array}{l}\text { ** Mêmes critères, mais couleur moins } \\
\text { uniforme, présence de tâches rou- } \\
\text { geâtres ou sanguines peu pronon- } \\
\text { cées. }\end{array}$ & & \\
\hline $2^{\mathrm{e}} \cdot \cdot \cdot \cdot$ & \multicolumn{3}{|c|}{$\begin{array}{l}\text { *** Tous les foies normalement engraissés mais ne répondant pas aux } \\
\text { critères ci-dessus énoncés à l'exception des foies de «volailles ». }\end{array}$} & \\
\hline $\begin{array}{l}\text { Volaille } \\
(\text { Poultry })\end{array}$ & Foies & non engraissés (Non fattened livers) & & \\
\hline
\end{tabular}
defects.

$\left.{ }^{*}\right)$ Smooth surface, white pinkish, uniform colour, without reddish spots, blood traces or apparent traces.

$\left({ }^{*}\right)$ Same criteria but less uniform colour, presence of little pronounced reddish spots or blood

(***) All normally fattened livers but not responding to the above mentioned criteria, foultry liver excepted.

\section{Méthodes d'analyse}

Une analyse statistique classique a permis d'obtenir les moyennes, variances et écarts-types par lot et tous lots réunis, ainsi que les coefficients de corrélation entre les divers paramètres. 
Des tests de $\chi^{2}$ ont permis de vérifier l'indépendance entre la classification des foies et les gaveurs ou les types génétiques.

Le modèle mathématique à effets fixés utilisé pour étudier la variation des différents caractères s'écrit :

$\mathrm{X}_{i j k l}=\mu+\mathrm{A}_{i}+\mathrm{B}_{j}+\mathrm{C}_{k}+(\mathrm{AB})_{i j}+(\mathrm{AC})_{i k}+(\mathrm{BC})_{j k}+\beta\left(\mathrm{Y}_{i j k l}-\mathrm{X} \ldots.\right)+\mathrm{E}_{i j k l}$

$\mathrm{X}_{i j k l}=$ mesure faite pour un caractère pour le $l^{\mathrm{e}}$ animal ayant eu une durée de gavage $\mathrm{Y}_{i j} k_{l}$, du $i^{\mathrm{e}}$ type génétique gavé par le $j^{\mathrm{e}}$ gaveur et dont le foie a été classé dans la $k^{\mathrm{e}}$ classe.

$\mu \quad=$ moyenne des $\mathrm{X}_{i j k l}$

$\mathrm{A}_{i}=$ effet fixé du type génétique $(i=\mathrm{I}$ à 4$)$.

$\mathrm{B}_{j}=$ effet fixé du gaveur $(j=\mathrm{I}$ et 2$)$.

$\mathrm{C}_{k}=$ effet fixé de la classe de foie $(k=\mathrm{I}$ à 4$)$.

$(\mathrm{AB})_{i j},(\mathrm{AC})_{i k},(\mathrm{BC})_{j k}=$ les 3 interactions entre les 3 facteurs 2 à 2 .

$\mathrm{E}_{i j k l}=$ effet résiduel aléatoire.

$\beta=$ coefficient de régression entre le caractère mesuré et la durée du gavage de l'animal.

$\mathrm{Y} \ldots . .=$ durée moyenne dı gavage.

Les coefficients de corrélation totaux et résiduels du modèle sont présentés. Les calcu1s ont été réalisés sur l'ordinateur MI'TRA I5.35 du Centre de Recherches I.N.R.A. de Toulouse.

\section{Résultats}

L'analyse de la variance réalisée par le modèle précédemment décrit a donné les résultats présentés dans le tableau 3. De nombreux effets sont plus fortement significatifs en partie dı fait de l'importance des effectifs considérés dans chacun des lots.

- L'effet souche est très hautement significatif sur l'ensemble des caractères mesurés. En ce qui concerne les critères d'élevage, cet effet provient d'une croissance significativement inférieure de la souche 22 (-364 $\mathrm{g}$ dès $53 \mathrm{j}$.) par rapport aux autres lots (fig. I). La souche II exprime ses potentialités à partir de 53 jours $(+220 \mathrm{~g}$ à $67 \mathrm{j}$.$) . Le croisement expérimental a un comportement similaire au$ croisement de référence. Les écarts de poids enregistrés pendant la période d'élevage se retrouvent pour les poids de carcasse et de paletot. Ceux-ci conduisent à un classement des lots d'étude : les deux types génétiques extrêmes (II et 22) sont significativement différents entre eux et les deux croisements ne se distinguent pas statistiquement. Pour le poids de foie frais, le croisement étudié (I2) est significativement supérieur aux autres types génétiques étudiés statistiquement identiques $(+46,18 \mathrm{~g}$ contre $-32,4 \mathrm{~g}$ au croisement de référence). Par contre, après étuvage à $55^{\circ}$ il semble perdre une partie de son avantage ( $29 \mathrm{~g}$ de plus que la perte moyenne), qui s'annule après étuvage à $\operatorname{IO}^{\circ}$ ( $45 \mathrm{~g}$ en dessous de la moyenne) au profit de la souche lourde (II). Le croisement de référence a un comportement inverse du croisement étudié puisqu'il récupère après étuvage à $105^{0}$ le handicap observé pour le poids de foie frais. 


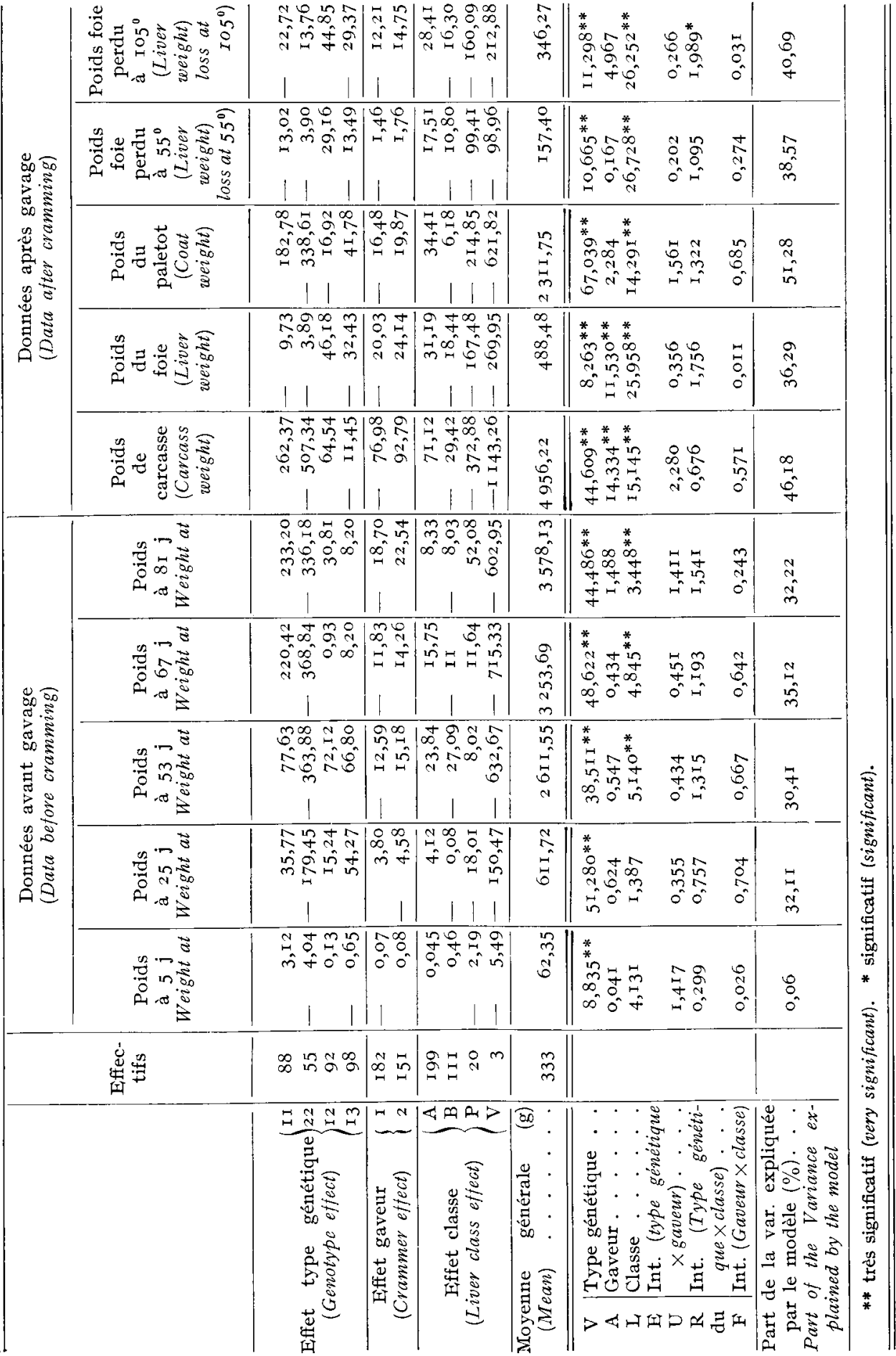




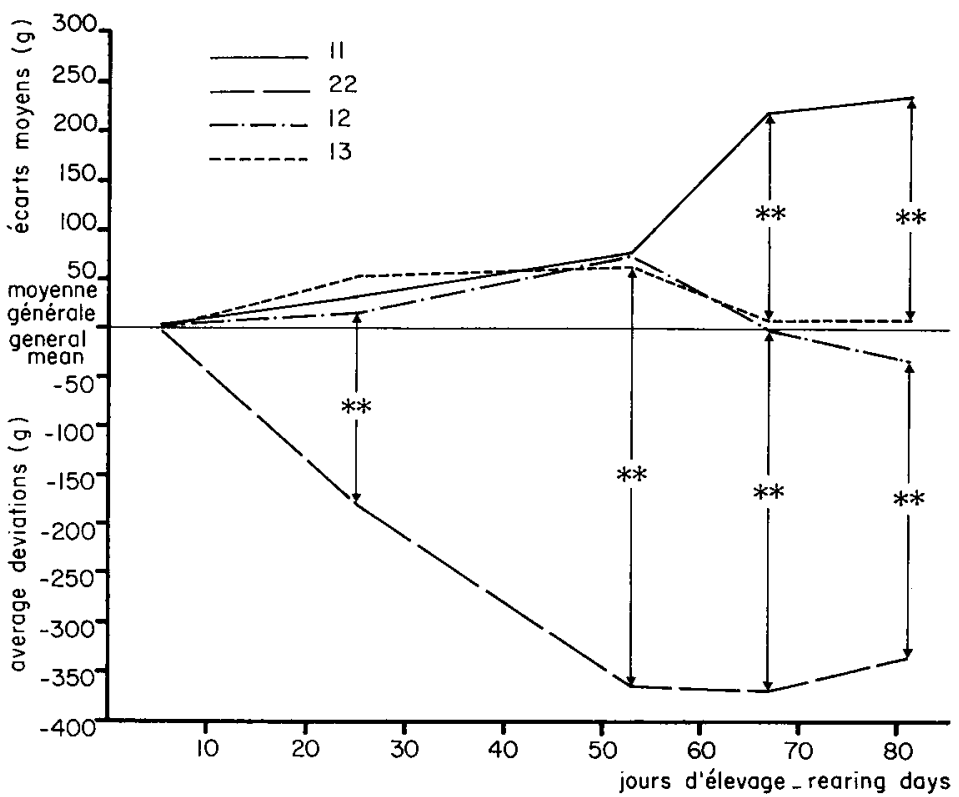

FIG. I. - Évolution des écarts-moyens (en g) à la moyenne générale du poids vif et de leur signification en fonction de l'âge, par type génétique.

Evolution of average deviations from general mean and significance according to age and genotype.

** significatif au seuil I p. Ioo.

* significatif au seuil $5 \mathrm{p}$. Ioo.

- I'effet gaveur non significatif sur les caractères mesurés avant gavage confirme que l'allotement a bien été réalisé de façon aléatoire entre gaveurs.

Le gaveur a un effet significatif sur les caractères mesurés après gavage, surtout pour le poids de carcasse et le poids de foie frais ( $44 \mathrm{~g}$ d'écart entre les deux). Il faut un étuvage à IO $5^{\circ}$ pour faire apparaître une fonte significativement supérieure des foies produits par le gaveur ayant obtenu les plus lourds.

- Dès 1'âge de 53 jours, dans notre expérience, le poids de l'animal est un critère discriminant de façon significative la qualité ultérieure du foie. Lres très petits animaux (pesant moins de $2 \mathrm{~kg}$ ) donneront des foies classés "volaille". Les poids enregistrés jusqu'à $8 \mathbf{I}$ jours ne peuvent rendre compte de la répartition dans les trois autres classes après gavage des animaux.

Après gavage, la classification du foie est directement liée aux différents poids enregistrés (carcasse, foie ou paletot). Les foies classés en " $A$ » ont 3I,I9 g de plus que la moyenne, ceux classés en " purée et volaille " I67 g et a70 g de moins respectivement.

Les mesures faites après étuvage à $55^{\circ}$ ou à $105^{\circ} \mathrm{C}$ montrent que les foies les mieux classés (donc les plus lourds) sont aussi les plus fondants.

- L'étude des interactions (significatives entre la souche et la classe du foie dans le seul cas de la fonte à I05 ${ }^{\circ}$ ) montre que pour les foies classés en purée, ceux de la souche de petit format (470 g perdus) ont fondu beaucoup plus que ceux de la souche lourde (230 g perdus).

Pour la plupart des caractères avant gavage et après abattage, le modèle utilisé explique entre 30 et $50 \mathrm{p}$. Ioo des différences observées. 


\begin{tabular}{|c|c|c|c|c|c|c|c|c|c|c|}
\hline 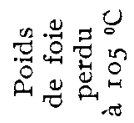 & $\begin{array}{l}\text { के } \\
\text { : } \\
0\end{array}$ & $\begin{array}{l}\underset{H}{ \pm} \\
0^{\circ}\end{array}$ & $\begin{array}{l} \pm \\
0 \\
0 \\
0^{\circ}\end{array}$ & $\begin{array}{l}\hat{D} \\
0 \\
0 \\
0\end{array}$ & $\begin{array}{l}\text { 今 } \\
\text { : } \\
0^{\circ}\end{array}$ & $\frac{m}{\tilde{y}}$ & 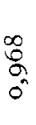 & $\begin{array}{c}n \\
\infty \\
0 \\
0\end{array}$ & $\begin{array}{l}\stackrel{0}{0} \\
0 \\
0\end{array}$ & $\begin{array}{l}\vec{n} \\
\stackrel{5}{\sigma} \\
\infty \\
0 \\
0\end{array}$ \\
\hline 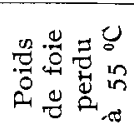 & $\begin{array}{l}\tilde{N} \\
\text { בै } \\
\text { - }\end{array}$ & $\begin{array}{l}\infty \\
\stackrel{\infty}{0} \\
0^{\prime}\end{array}$ & $\stackrel{\sigma}{\sigma}$ & $\begin{array}{l}\hat{m} \\
\text { ?y } \\
\text { ó }\end{array}$ & 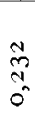 & 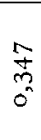 & $\stackrel{i}{\vec{S}}$ & $\frac{8}{2}$ & 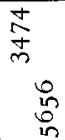 & $\begin{array}{c}\hat{n} \\
\infty \\
0 \\
0\end{array}$ \\
\hline
\end{tabular}

\begin{tabular}{|c|c|c|c|c|c|c|c|c|c|}
\hline 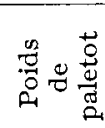 & $\underline{n}$ & $\stackrel{m}{2}$ & $\begin{array}{l}12 \\
12\end{array}$ & $\ddot{8}$ & $\begin{array}{l}\infty \\
\stackrel{0}{0} \\
0\end{array}$ & $\stackrel{N}{\infty}$ & $\begin{array}{l}\text { in } \\
\text { in } \\
0\end{array}$ & 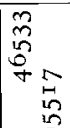 & $\begin{array}{l}\vec{N} \\
\text { ? }\end{array}$ \\
\hline
\end{tabular}

\begin{tabular}{|c|c|c|c|c|c|c|c|c|c|c|}
\hline$\stackrel{0}{0}$ & $\begin{array}{l}0 \\
\text { in } \\
\text { "n }\end{array}$ & $\underset{m}{m}$ & 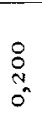 & 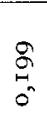 & $\begin{array}{l}\infty \\
\text { Na } \\
\text { on }\end{array}$ & $\begin{array}{l}m \\
\text { sn } \\
0\end{array}$ & 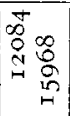 & $\begin{array}{c}\stackrel{+}{3} \\
3 \\
0 \\
0\end{array}$ & $\begin{array}{l}\vec{N} \\
\text { D } \\
0\end{array}$ & $\begin{array}{l}\tilde{2} \\
\hat{\sigma} \\
0\end{array}$ \\
\hline
\end{tabular}

\begin{tabular}{|c|c|c|c|c|c|c|c|c|c|c|}
\hline 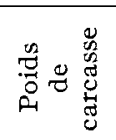 & $\begin{array}{l}\hat{N} \\
\text { Na } \\
0^{n}\end{array}$ & $\begin{array}{l}\frac{H}{m} \\
0^{2}\end{array}$ & $\begin{array}{l}0 \\
\infty \\
12 \\
0\end{array}$ & $\begin{array}{l}\hat{0} \\
0_{0}^{\prime}\end{array}$ & 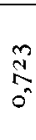 & 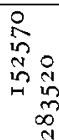 & $\begin{array}{l}\infty \\
0 \\
0 \\
0 \\
0\end{array}$ & $\begin{array}{l}\infty \\
\vdots \\
a \\
0\end{array}$ & $\begin{array}{l}0 \\
0 \\
0 \\
0 \\
0\end{array}$ & $\begin{array}{l}\infty \\
\infty \\
+ \\
0\end{array}$ \\
\hline
\end{tabular}

\begin{tabular}{|c|c|c|c|c|c|c|c|c|c|c|}
\hline 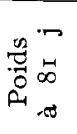 & $\begin{array}{c}\text { D } \\
\text { M } \\
\text { - }\end{array}$ & $\begin{array}{l}\text { in } \\
\text { ma }\end{array}$ & $\begin{array}{l}\text { 点 } \\
\mathrm{o}^{\prime}\end{array}$ & 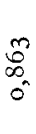 & 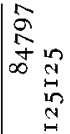 & $\begin{array}{l}\stackrel{1}{0} \\
0^{n}\end{array}$ & 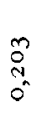 & 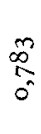 & $\begin{array}{l}\stackrel{8}{\circ} \\
\stackrel{-}{\circ}\end{array}$ & $\begin{array}{l}\stackrel{m}{\oplus} \\
\stackrel{0}{0}\end{array}$ \\
\hline
\end{tabular}

\begin{tabular}{|c|c|c|c|c|c|c|c|c|c|c|}
\hline$\stackrel{\infty}{0}$ & $\stackrel{8}{\circ}$ & $\begin{array}{l}\hat{\hat{v}} \\
\text { } \\
\hat{0}\end{array}$ & 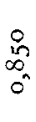 & $\begin{array}{l}\frac{\pi}{4} \\
\text { in } \\
\text { in } \\
\frac{y}{4} \\
\end{array}$ & $\begin{array}{l}\infty \\
: \\
0 \\
0\end{array}$ & 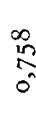 & $\begin{array}{l}0 \\
\text { n1 } \\
1 \\
0\end{array}$ & $\underset{0}{\stackrel{N}{N}}$ & 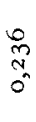 & $\begin{array}{l}\stackrel{+}{5} \\
0^{\prime}\end{array}$ \\
\hline
\end{tabular}

\begin{tabular}{|c|c|c|c|c|c|c|c|c|c|c|}
\hline 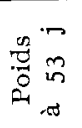 & $\begin{array}{l}2 \\
2^{2} \\
0\end{array}$ & $\begin{array}{l}\text { 今. } \\
0 \\
0\end{array}$ & 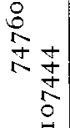 & $\begin{array}{c}\mathbb{R} \\
\infty \\
0^{-}\end{array}$ & $\begin{array}{l}\infty \\
\text { ra } \\
0^{\prime}\end{array}$ & $\begin{array}{l}8 \\
8 \\
\mathscr{B}_{0}\end{array}$ & 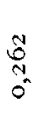 & $\begin{array}{l}D^{2} \\
0 \\
0\end{array}$ & $\begin{array}{l}\text { wn } \\
\hat{n} \\
0 \\
0\end{array}$ & $\begin{array}{l}0 \\
\stackrel{1}{1} \\
0\end{array}$ \\
\hline
\end{tabular}

\begin{tabular}{|c|c|c|c|c|c|c|c|c|c|c|}
\hline 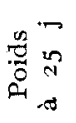 & $\begin{array}{l}m^{\infty} \\
0_{0} \\
0\end{array}$ & 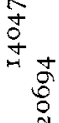 & 究 & $\begin{array}{l}\text { m } \\
\text { d } \\
0\end{array}$ & $\begin{array}{l}8 \\
i^{2} \\
0\end{array}$ & $\begin{array}{l}5 \\
\text { in } \\
0 \\
0\end{array}$ & $\begin{array}{l}\text { in } \\
\text { ?n } \\
0\end{array}$ & $\begin{array}{l}\text { Â } \\
\text { Wh } \\
\sigma^{2}\end{array}$ & $\begin{array}{l}R \\
0_{0}^{\prime}\end{array}$ & $\begin{array}{l}8 \\
8 \\
0 \\
0\end{array}$ \\
\hline
\end{tabular}

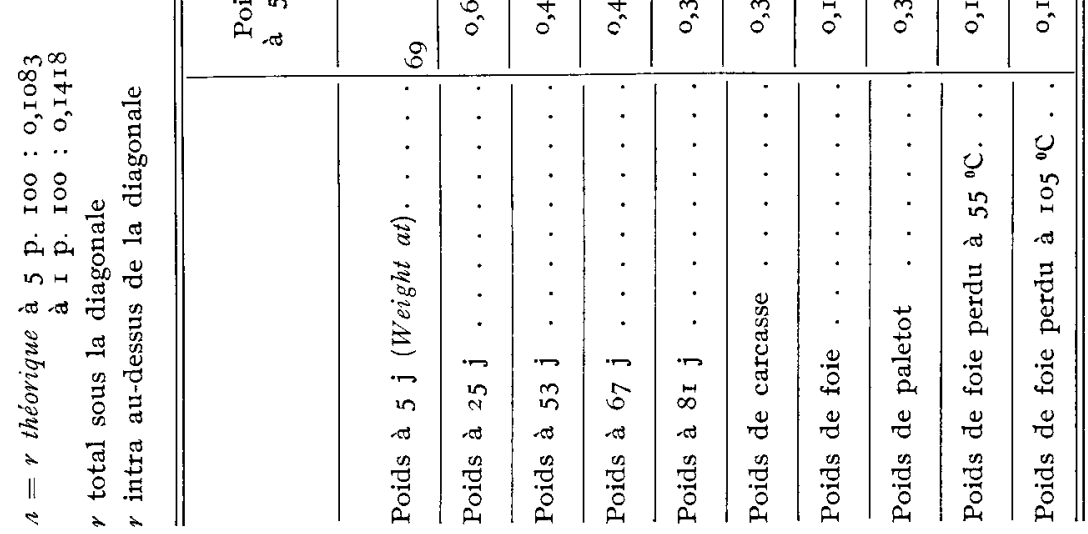


- Le tablean 4 présente en dessous de la diagonale les corrélations totales entre caractères et au-dessus de la diagonale celles calculées après élimination des effets pris en compte par le modèle, celles-ci sont légèrement inférieures aux précédentes. Ceci procède de la variabilité entre souches qui est très supérieure pour les poids notamment à celle intra souche (présence dans l'expérience de deux souches extrêmes). Les corrélations entre les poids à différents âges varient de 0,9 à 0,2 ; elles sont d'autant plus faibles que les poids deux à deux sont éloignés. Le poids de la carcasse est plus lié au poids du paletot $(0,9)$ qu'au poids du foie $(0,5)$. La fonte est très corrélée au poids du foie $(0,97)$.

Cette corrélation est d'autant plus forte que l'étuvage a été fait à une température plus élevée.

\section{Discussion}

Cette expérience souligne l'intérêt du canard de Barbarie mâle pour la production de foie gras. Les résultats moyens de gavage obtenus (488 g) sont voisins (De CARVILIE et De CroutTe, I978), voire supérieurs (IECLERCQ et al., I973) à ceux observés sur le canard Mulard qui est classiquement utilisé à cette fin. Notre étude a pour but de comparer les potentialités de souches de Barbarie commercialisables (décrites par WEHRUNG, I977) dont l'intérêt réside dans l'adéquation des qualités d'élevage et d'engraissement;

- L'étude des poids successifs pendant la période d'élevage (5 à $8 \mathrm{I} \mathrm{j}$ ) conformes à ceux observés pour l'espèce (LECLERCQ et De CARVILLE, I976 et I979) confirme, à un degré moindre pour la souche de petit format, les potentialités recherchées pour la production de chair $(3,6 \mathrm{~kg}$ à $8 \mathrm{I} \mathrm{j}$. pour les croisements mis en comparaison). Les écarts enregistrés avec la bibliographie qui rapporte des poids à $\mathrm{I} 2$ semaines compris entre 3 et $4 \mathrm{~kg}$ sont sans doute dus à des variations dans le régime alimentaire ou de conditions d'ambiance (LECLERCQ et De CARVILLE, I975).

- L'étude des corrélations intra-types génétiques entre les poids avant gavage et les critères de classification économique montre, parmi les poids mesurés pendant la phase de croissance, 1'intérêt du poids à 53 jours : il semble qu'à partir de cet âge, dans nos conditions expérimentales, les animaux les plus lourds donneront des foies de meilleure qualité et de poids plus élevé présentant toutefois l'inconvénient d'une perte à l'étuvage plus importante. Ceci doit être pondéré par le fait du peu d'effectif de foies classés en "purée et volaille " que nous avons eu dans notre expérimentation. Un poids vif de moins de $2 \mathrm{~kg}$ pour un mâle de cet âge-là rend sans doute plus compte d'un mauvais état de santé que du potentiel de l'animal.

- La corrélation intra-type génétique entre poids du foie et poids à 53 jours de 0,2 paraît supérieure à celle rapportée pour un stade équivalent chez l'oie par Nicoras (I977) qui cite des valeurs de o, Io à $0, I_{5}$; ceci pourrait présenter un certain intérêt expérimental pour une sélection précoce en vue de la production de foie puisque, selon ColonNa CESARI (I973), le poids à 8 semaines chez le canard de Barbarie serait aisément sélectionnable (héritabilité 0,6 ). Les corrélations phénotypiques mesurées deux à deux entre les différents critères (poids à 2 mois, poids de mise en gavage, poids de carcasse, poids du foie) sont analogues à celles mesurées sur l'oie par MALLARD (I975) à partir des résultats enregistrés à la station d'Artiguères. 
- L'effet gaveur sur les poids de carcasse et de foie a déjà été mis en évidence par ailleurs sur l'oie (BLUM et al., I97I; MALIARD, 1975) et sur le mulard (DuBoIs, I 980 ) où l'on rencontre pour les mêmes types génétiques, des poids moyens de foie allant du simple au double selon le gaveur. Dubors (I980) dans son analyse des facteurs de variation du poids du foie et de la carcasse montre que cet effet est prépondérant par rapport aux facteurs souche et éleveur. Il semble, d'après les observations que nous avons pu réaliser, que cet effet est essentiellement dût à la quantité journalière de maïs que le gaveur fait absorber aux animaux et à la vitesse d'augmentation de cette quantité. Contrairement à ce que l'on a longtemps affirmé (FLEURET, I953), c'est le gaveur qui a apporté les quantités journalières les plus élevées qui a eu les poids de foie les plus élevés, la durée de gavage la plus courte, mais aussi les fontes les plus importantes. L'analyse des résultats de consommation pour les différents lots du protocole expérimental décrit ici fera l'objet d'une autre publication.

- Le croisement expérimental (type génétique I2) se révèle d'un intérêt certain pour la production de foie gras, bien qu'il soit sans doute pénalisé par la mauvaise aptitude des foies gras à l'étuvage. Ce dernier point, consécutif au lien étroit entre le poids du foie et la fonte (corrélation de 0,97 à I0 $5^{\circ}$ ) doit être pondéré par l'imprécision de l'extrapolation qui a été faire pour estimer la fonte qu'aurait eu le foie entier : l'estimation a été faite à partir d'un très petit échantillon (environ $4 \mathrm{~g}$ ). Des mesures analogues ont été réalisées par Nir et NITSAN (I976) à partir de petits échantillons dont la fonte est fortement correlée avec celle du foie entier chez 1'oie. Elles ont, en outre, montré que la congélation n'avait pas d'effet significatif sur la fonte.

D'un point de vue génétique, on enregistre un effet d'hétérosis entre les souches I I et 22 de II p. IOO pour le poids du foie, qui n'est plus que de 5 p. Ioo pour le poids après étuvage à $55^{\circ}$ et de $2 \mathrm{p}$. Ioo après étuvage à $105^{\circ}$. Il semble que la combinaison d'une souche lourde (type génétique II) et d'une souche de petit format (type génétique 22) permet de pallier les inconvénients que possède chacune des races pures pour le poids du foie frais. La souche lourde bien adaptée à la production de chair produit des foies moyens car, à 1'âge où elle est gavée, son degré de maturité est faible; or, nous savons, pour le canard (LECLERCQ et De CARVILLE, I 978), comme pour les autres espèces que c'est en fin de développement que se fait la lipogénèse. Par contre, une souche de petit format ferait ses dépôts graisseux plus tôt mais le poids de foie est légèrement pénalisé du fait de son petit format.

L'avantage résultant de l'hétérosis entre les deux souches s'estompe après les étuvages successifs pour conduire à un poids de foie final encore légèrement supérieur à celui issu du croisement de référence ( $143,5 \mathrm{~g}$ contre $139, \mathrm{I} 6 \mathrm{~g}$ ).

\section{Conclusion}

L'animal croisé mis en testage dans cette expérience semble bénéficier des avantages des deux souches (poids d'organes et précocité) en évitant un certain antagonisme qui semble apparaitre entre-souche pour les poids des foies gras et la croissance. Ceci pose le problème de la sélection pour augmenter le poids du foie, 1'idéal étant d'obtenir des animaux qui, à l'âge de $8 \mathrm{I}$ jours, sont relativement matures et présentent un potentiel de croissance élevé. Ces constatations laissent entrevoir des études permettant d'apprécier le stade auquel le gavage 
sera le plus efficace en fonction du degré de maturité des animaux. Un véritable mode d'emploi de chacune des souches devrait être mis au point malgré les difficultés de réalisation que ceci peut entraîner, tant au niveau des éleveurs que des gaveurs; l'effet sur les performances de foie devrait être approfondi. L,e problème de la fonte devrait aussi être étudié dans le même sens, notamment en prenant en compte l'effet de la durée de gavage sur ce critère. L'objectif du sélectionneur doit être de constituer des types génétiques (sans doute des croisements entre des souches sélectionnées sur le poids à 8 semaines et des souches précoces), à fort potentiel de production de foie utilisées dans les organisations d'élevage et de gavage rationnalisées.

Accepté pour publication en septembre 1980.

\section{Remerciements}

Nous remercions et assurons de toute notre amitié M. POUJARDiEu B. (S.A.G.A. I.N.R.A). pour l'aide qu'il nous a apportée dans le traitement et l'interprétation de nos données.

\section{Summary}

Fat liver production of male Muscovy ducklings : analysis of some factors of variance.

An experiment was made to compare different genotypes of male Muscovy ducklings (two pure breeds, their cross and a commercial line as control) for fat liver production. All the birds were reared simultaneously and controlled at different ages up to $\mathrm{r} 2 \mathrm{weeks}$ and after cramming in the same place with two crammers. The statistical analysis of data shows a significant effect of genotype on each trait, a significant effect of the crammer on the economical traits after cramming and a positive relationship between the liver class and the bird weight at 8 weeks of age. The experimental cross led to slightly improved values for carcass and liver traits. This new cross is still penalized by the high significant correlation between the liver weight and its melting losses when cooked. These results suggest the necessity of defining more accurately the mode of utilisation of each strain with the aim of improving their profit earning capacity.

\section{Références bibliographiques}

BABILE R., I980. L'expérimentation sur 1'utilisation du canard de Barbarie pour la production de foie gras. Coteaux de Gascogne, janvier $1980,3^{6-3^{8}}$

Blum J. C., Monacion G., Leclerce B., I97I. Liver steatosis of force-fed geese as influenced by the protein level of the diet. Acta Vet. Acad. Sci. Hung., 21, 307-3I I.

Colonna-CESARI M., 1973- La sélectiont du canard de Barbarie. Session canard I.T.A.V.I., 8-9 février 1973, I-13.

De Carvilie H., DE Croutte A., I978. In "Le canard». Ed. Vigot, Paris, pp. 232.

Dunors J. P., I980. Production du foie gras. Les rôles de l'éleveur et du gaveur de canards mulards sont déterminants. Courvier Avicole $\mathrm{n}^{\mathrm{b}} 774,28-29$.

FARRAN M. T., I978. Utilisation du canard de Barbarie pour la production de palmipèdes gras. Mémoive de D.E.A., pp. I Io.

Fleuret P. H., I953. La production du foie gras de volaille destiné à la consommation. $A n n$. Nutr. Alim., 7, C 97 . 
Leclerco B., Hassan I., Thivend D., Blum J. C., I973. Utilisation comparée du maîs entier et du maïs broyé pour le gavage du cariard. Ann. Zootech., 22, 337-340.

LEClERCQ B., De Carville H., 1975. Besoin en protéines du caneton de Barbarie entre les âges de 4 et I I semaines. Ann. Zootech., 24, 2 I 7-227.

LECLERQ B., De CARVILLE H., I976. L'alimentation azotée du caneton de Barbarie : étude du besoin du caneton mâle entre les âges de 4 et I 2 semaines. Ann. Zootech., 25, I89-I97.

LECLERCQ B., De CARVIL entre les âges de 8 et I 2 semaines. Ann. Zootech., 27, I-7.

Lecherce B., De Carvilie H., 1979. Les besoins en phosphore du caneton de Barbarie. Ann. Zootech., 28, IOI-107.

MALI.ARD Y., I975. Étude des performances de gavage chez l'oie. Mémoire de fin d'études E.N.S.A., Toulouse, pp. 40

Nicolas A., 1977. Influence du jars sur les performances de reproduction et de gavage. Mémoire de fin d'études E.N.S.A. Toulouse, p. I50.

NiR I., Nitsan $Z$., I976. Goose fatty liver composition as related to the degree of steatosis, nutritional and technological treatments, and a simplified method for quality estimation. Ann. Zootech., 25, 46r-47o.

Pavaux C., Jolly A., I 968 . Note sur la structure vasculo-canaliculaire du foie des oiseaux domestiques. Rev. Méd. Vét., 119, 445-466.

Wehrung F., 1977. Le canard de Barbarie progresse. L'élevage Grimaud Frères aussi. Courrier Avicole $\mathrm{n}^{\mathrm{b}} 673,28-33$. 